\title{
Liberté et institution : Sur la phénoménologie de l'Einbildungskraft dans la pensée de Marc Richir
}

\author{
Par TETSUO SAWADA
}

Université de Toyama

Résumé Le présent travail vise à mettre en lumière le statut de l'« imagination (Einbildungskraft) » kantienne dans la phénoménologie. Pour accomplir cette tâche, il convient selon nous de se référer aux travaux de Marc Richir, tels que l'article "L'origine phénoménologique de la pensée » et l'ouvrage Phénoménologie et institution symbolique. Car, en discutant soigneusement de l'imagination kantienne dans la Critique de faculté de juger, ce phénoménologue tente de dégager, à partir du concept de l'imagination, le caractère paradoxal de la vie de la conscience humaine. Il s'agit alors d'une « imagination» dédoublée à son origine entre la «liberté phénoménologique » et $l^{\prime}$ ' institution symbolique » de la Raison. La première nous permet de voir la phénoménalité des phénomènes et leur phénoménalisation dans la vie de la conscience humaine. La dernière a pour effet de stabiliser, et même d'aplatir, à l'insu du soi, la première. Ainsi, les phénomènes ne se phénoménalisent qu'en étant menacés par la crise de leur disparition dans les idées de la Raison. Cela revient à dire que, pour ce phénoménologue profondément inspiré par l'« imagination » kantienne, la phénoménologie est fondée foncièrement à la limite entre ce qui est phénoménologique («liberté phénoménologique ») et ce qui est non phénoménologique (la Raison). C'est, dès lors, par son approche phénoménologique qu'on peut voir la contribution de l'« imagination » kantienne à la phénoménologie transcendantale.

\section{Introduction}

L'un des caractères majeurs de la philosophie transcendantale, que ce soit l'idéalisme transcendantal ou la phénoménologie transcendantale, consiste, 
sans doute, à dévoiler la mise en jeu spécifique de l'« imagination (Einbildungskraft) » dans les activités humaines ${ }^{1}$. Kant, dans sa première critique, découvre le rôle intermédiaire que joue l'Einbildungskraft entre la sensibilité (l'intuition) et l'entendement (le concept); le même auteur, dans sa troisième critique, propose la mise en jeu spécifique de l'Einbildungskraft. Par ailleurs, Husserl souligne la puissance de la "phantasia (Phantasie) », et son fonctionnement au cours de la réduction eidétique, comme moyen d'accéder à l'essence (ou l'eidos) des vécus intentionnels.

En envisageant ainsi la pluralité de caractères et de généalogies de l' ' imagination » dans la tradition de la philosophie transcendantale, il sera nécessaire de poser deux questions : de quelle façon et dans quelle mesure l'Einbildungskraft et sa puissance spécifique, telles qu'elles sont proposées par Kant, peuvent-elles trouver leur statut dans la phénoménologie ? En outre, quels sont le statut et les potentialités de l'Einbildungskraft dans la phénoménologie contemporaine?

Pour répondre à ces questions, les ouvrages et les articles de Marc Richir méritent d'être mentionnés. Car ce phénoménologue, récemment disparu, ne cessait de discuter de l'Einbildungskraft dans la troisième critique kantienne et en dégageait les caractères phénoménologiquement bien spécifiques. C'est dans cet horizon que le présent travail se donne comme tâche de discuter de l'approche richirienne de l'Einbildungskraft.

\section{Einbildungskraft phénoménologique selon Richir}

\subsection{Le jugement réfléchissant et l'imagination selon Kant}

Étant donné que, dans la troisième critique kantienne, l'« imagination »s'attache strictement à un acte de juger bien particulier du sujet, nommé par Kant « jugement réfléchissant», il nous faudra aborder celui-ci avant de pénétrer dans celle-là et sa lecture richirienne. Kant le définit en disant : « Si seul le particulier est donné, et si la faculté de juger doit trouver l'universel qui lui

\footnotetext{
${ }^{1}$ Le présent travail est la version remaniée d'un texte exposé le 26 avril 2016 au $10^{\mathrm{e}}$ Séminaire annuel, organisé par l'Unité de recherche «Phénoménologies » de l'Université de Liège sur le thème de «L'acte d'imagination: Approches phénoménologiques ». Avant de commencer la discussion, nous tenons à exprimer nos sentiments de profonde gratitude à Maud Hagelstein, Alievtina Hervy, Bruno Leclercq et aux autres organisateurs qui nous ont accueilli dans une ambiance chaleureuse et un fort esprit de recherche. La rédaction du texte est soutenue par JSPS KAKENHI (Grant-in-Aid for Young Scientists (B), Grant Number 15K16604).
} 
correspond, elle est simplement réfléchissante ${ }^{1}{ }^{2}$. Lorsqu'un jugement est « réfléchissant», l'acte de juger du sujet ne présuppose pas encore la loi « universelle » qui pourrait le subsumer sous tel ou tel concept prédéterminé, mais ne fait qu'y aspirer dans son mouvement de réflexion.

Selon Kant, la spécificité de ce jugement est précisée dans le «jugement de goût» qui concerne notamment l'expérience du «beau »: «Soit cette rose que je vois; par un jugement de goût j'affirme qu'elle est belle $»^{2}$. Dans cette proposition, « cette rose est belle », le prédicat « beau (belle) » est prononcé immédiatement; pour le figurer, ce jugement n'a pas besoin d'avoir recours aux processus logiques (syllogisme) ni aux déterminations objectives de la chose (la taille, la couleur, etc. de la « rose $»)^{3}$. Tout cela signifie que, lorsque le jugement de goût est mis en jeu dans la vie humaine, l'homme ne s'intéresse plus au concept analytique de la chose mais uniquement à son caractère spécifique (le prédicat: «belle »). C'est ainsi que la seule loi conforme à ce jugement est la «loi de spécification (Gesetz der Spezifikation) $»^{4}$. Néanmoins, selon l'explication kantienne, ce jugement est destiné, finalement, à être validé «pour tout le monde (für jedermann) $»^{5}$. Cela revient à dire que, sans se limiter au jugement privé et personnel, il amène le sujet qui prononce le prédicat à entrer en accord avec d'autres, sans procédés intermédiaires tels que la logique formelle, les concepts, les déterminations objectives ou les règles sociales.

Selon la troisième critique kantienne, c'est au sein de ce jugement singulier et spécifique que l'imagination (Einbildungskraft) est mise en jeu dans la vie humaine ; plus précisément, Kant appelle sa mise en jeu «appré-

${ }^{1}$ E. Kant, Kritik der Urteilskraft, 1790, cité d'après la version de la Königlich Preußischen Akademie der Wissenschaften (Ak.), Ak. V, p. 180 ; trad. fr. A. Philonenko, Critique de la faculté de juger, Paris, J. Vrin, 1993, p. 40.

${ }^{2}$ Ibid., p. 215, trad. fr., p. 78.

3 Notre discussion sur la théorie kantienne du «jugement réfléchissant» doit beaucoup à la remarque d'Hannah Arendt dans ses cours sur la théorie kantienne du politique. En mentionnant le même exemple kantien, elle dit à juste titre : « Si l'on dit "Quelle belle rose !", on ne parvient pas à ce jugement en énonçant d'abord "Toutes les roses sont belles, cette fleur est une rose, donc cette rose est belle." $\mathrm{Ni}$, inversement, "le Beau, ce sont les roses, cette fleur est une rose, donc elle est belle" »(H. Arendt, Lectures on Kant's Political Philosophy, edited and with an interpretive essay by R. Beiner, The University of Chicago Press, 1982, p. 13-14; trad. fr. M. Revault d'Allonnes, Juger. Sur la philosophie politique de Kant, Paris, Seuil, 1991, p. 31).

${ }^{4}$ E. Kant, Kritik der Urteilskraft, Ak. V, p. 186, trad. fr., p. 47.

${ }^{5}$ Ibid., p. 216, trad. fr., p. 78. 
hension des formes dans l'imagination (Einbildungskraft)» (ibid., 190/52). L'imagination mise en jeu dans le jugement réfléchissant permet au sujet de saisir immédiatement la «forme» de la chose qu'il perçoit, de façon à ce qu'il n'ait aucun besoin d'avoir recours aux processus qui pourraient la déterminer dans tel ou tel concept correspondant. L'activité du sujet se met alors à entrer entièrement dans un « jeu » :

L'universelle communicabilité subjective du mode de représentation dans un jugement de goût, devant se produire sans présupposer un concept déterminé, ne peut être autre chose que l'état d'esprit dans le libre jeu de l'imagination et de l'entendement (der Gemütszustand in dem freien Spiele der Einbildungskraft und des Verstandes $)^{1}$.

Kant indique que l'imagination amène l'«entendement» au «jeu». Cela revient à dire que celui-ci empêche le sujet, et sa faculté cognitive fondée sur l'entendement, de juger de son objet suivant tel ou tel « concept déterminé ». Au lieu de faire correspondre son acte de juger à telle ou telle catégorie correspondante, son état d'esprit (Gemütszustand) est alors affecté et invité au libre jeu. L'imagination a ainsi pour rôle, dans la troisième critique kantienne, 1) de suggérer à l'entendement humain des variations dans le jeu et 2) de mettre en mouvement l'esprit humain dans une affectivité (Gemüt).

\subsection{L'accès à la phénoménalité du phénomène}

Les notions kantiennes - le jugement réfléchissant, l'imagination et son libre jeu — dans la troisième critique permettent à Richir d'élaborer la phénoménologie transcendantale dans une nouvelle perspective. Tout cela apparaît dans son article intitulé «L'origine phénoménologique de la pensée » (1984). L'auteur y écrit que " dans le goût est mis hors circuit, de manière quasi husserlienne, tout intérêt en l'existence (Existenz) (ou la nonexistence) de l'objet $»^{2}$. Dans le jugement réfléchissant, l'activité du sujet est elle-même indifférente à la position et à l'existence des choses dont il juge. Selon l'interprétation ci-dessus, la manière d'être du sujet imaginant peut être rapprochée conceptuellement de la «mise hors circuit (Ausschaltung)» au sens de la phénoménologie husserlienne. Husserl l'explique: "Tandis qu'elle [la thèse] demeure en elle-même ce qu'elle est, nous la mettons pour

${ }^{1}$ Ibid., p. 217-218, trad. fr., p. 81.

${ }^{2}$ M. Richir, "L'origine phénoménologique de la pensée », La liberté de l'esprit, $\mathrm{n}^{\circ}$ 7, Paris, Balland, 1984, p. 67. 
ainsi dire "hors de jeu (ausser Aktion)", "hors circuit (schalten aus)", "entre parenthèses (klammern ein)" ${ }^{1}$. La «mise hors circuit» exclut telles ou telles thèses, soit physiques, soit psychologiques, sur lesquelles la connaissance humaine prendrait appui avec son attitude naïve et naturelle. Pourtant, Husserl ne la considère jamais comme semblable au «doute » cartésien ou à une «négation » sceptique. Car elle a pour effet de présenter le " vécu » ${ }^{2}$ qui dévoile la structure intentionnelle de la conscience.

C'est dans ce contexte que Richir met en relation le jugement réfléchissant kantien avec la "mise hors circuit » husserlienne. Tout cela signifie que, sans se limiter à la spécification de l'objet du jugement, la théorie kantienne du «jugement réfléchissant» n'est, pour Richir, rien d'autre que l'accès au phénomène. C'est ainsi que, dit l'auteur, le jugement réfléchissant est «un jugement portant sur le phénomène en tant que phénomène immédiatement rapporté à sa phénoménalité $»^{3}$. Si le jugement en tant que « jugement réfléchissant» a pour nature d'échapper aux concepts prédéterminés, sa force spécifique nous permet, sur le plan phénoménologique, de se mettre à l'écart de l' « attitude naturelle » dans laquelle notre conception du monde est naïvement déterminée. C'est pour cette raison que le jugement réfléchissant nous permet de s'approcher immédiatement du "phénomène », à savoir du "phénomène immédiatement rapporté à sa phénoménalité ». On pourrait dire que la lecture richirienne de la troisième critique consiste ainsi à trouver dans le fonctionnement du "jugement réfléchissant» les idées inspirant la manière phénoménologique de s'approcher de la phénoménalité du phénomène, c'est-à-dire l'accès immédiat au "phénomène ».

\subsection{L'imagination phénoménologique et la phénoménalisation des phéno- mènes}

Si le jugement réfléchissant est un accès à la phénoménalité du phénomène, on pourrait considérer que l' ' imagination » fonctionnerait, dans la phénoménologie richirienne, comme fondement de l'accès à la phénoménalisation de ce phénomène. En effet, l'auteur précise, toujours dans le même article,

\footnotetext{
${ }^{1}$ E. Husserl, Ideen zu einer reinen Phänomenologie und phänomenologischen Philosophie. Erstes Buch: Allgemeine Einführung in die reine Phänomenologie (1913), Halle, Max Niemeyer, cité d'après Husserliana Band III, p. 63; trad. fr. Paul Ricoeur, Idées directrices pour une phénoménologie pure et une philosophie phénoménologique, Paris, Gallimard, coll. « Tel », 1950, p. 99.

${ }^{2}$ Ibidem.

${ }^{3}$ M. Richir, «L'origine phénoménologique de la pensée », art. cit., p. 67-68.
} 
l'importance phénoménologique de l'imagination. Tout cela est abordé dans le contexte du «sens commun (Gemeinsinn)» dans la troisième critique kantienne. La particularité de l'interprétation richirienne du " sens commun » consiste en ceci que l'auteur en dégage la forme primordiale de la relation intersubjective, tandis que Kant le discute en vue d'en dégager la Raison pratique ou «Sollen» : « chacun doit (Sollen) admettre [notre jugement] ${ }^{1}$. Richir appelle "quasi sauvage" cette forme primordiale de la relation intersubjective au moment de « sens commun» :

Que ce quasi sauvage soit éprouvé sans concept comme universellement communicable et nécessaire, cela signifie donc qu'en un sens il constitue l'entrée même de l'homme au monde comme monde commun, par suite l'entrée même de l'homme en son humanité, et de là, la part de l'homme en droit antérieure à ce qui se réfléchit comme l'institution sociale et historique, toujours déjà réglée, en quelque sorte, depuis elle-même, selon l'ordre symbolique qui constitue le nœud même de l'institution ${ }^{2}$.

En réfléchissant sur l'opération du «sens commun », Richir souligne moins son aspect moral (Sollen) que son aspect phénoménologiquement intersubjectif. C'est à travers lui qu'un homme se met à entrer en communication avec d'autres. En outre, étant fondée sur le jugement de goût, cette communication se fait de telle façon que sa forme ne présupposerait aucune loi sociale ou morale ni code rituel : en somme, au sein du fonctionnement du « sens commun », elle se dérobe à tous les types de l'« institution sociale ». C'est pour cette raison que, à l'instar de la terminologie du dernier MerleauPonty, Richir définit cette forme primitive de la relation intersubjective comme « quasi sauvage » et non « sociale».

Le statut phénoménologique de l'imagination peut être repéré dans ce contexte du «sens commun» en tant que «quasi-sauvage». Car Richir explique la manière dont elle y est mise en jeu :

Ensuite et corrélativement, le phénomène en tant que matrice symbolique il ne s'agit pas encore du symbole socialement institué —, à savoir en tant qu'élément rassemblant les «formations» de l'imagination comme «représentations parentes » s'étendant «à perte de vue », ne joue ce rôle que par

\footnotetext{
${ }^{1}$ E. Kant, Kritik der Urteilskraft, Ak. V, p. 239, trad. fr., p. 111.

${ }^{2}$ M. Richir, «L'origine phénoménologique de la pensée », art. cit., p. 72.
} 
rapport à d'autres phénomènes non actuellement présents dans le phénomène, mais à l'œuvre, latéralement, en absences, ou par éclats et fragments ${ }^{1}$.

L'auteur affirme ici une certaine puissance, inhérente elle-même à l'imagination, de se transférer et de "s'étendre » à d'autres phénomènes («autres phénomènes non actuellement présents ») avec lesquels l'individu n'imaginait jamais avoir de rapports possibles. Comme on l'a vu, Kant affirme que l'imagination est un « jeu » et que celui-ci déplace l'entendement humain des concepts déterminés vers la liberté qui affecte son esprit. Si on explique tout cela à partir de l'interprétation richirienne de l'imagination, on peut dire que c'est à travers cette force de jeu ${ }^{2}$ que les phénomènes dans lesquels le sujet éprouve et vit se mettent à s'ouvrir à «d'autres phénomènes » sans pouvoir être fermés sur chacun de leurs caractères individuels : « un phénomène ne se phénoménalise que s'il se phénoménalise du même coup pour un autre phénomène $»^{3}$. Dans la phénoménologie richirienne, si l'imagination est mise en jeu au sein du « sens commun », ce n'est pas seulement parce qu'un homme devient capable de communiquer avec d'autres dans la communauté humaine, mais aussi parce que les phénomènes desquels il s'approche se transposent en d'autres sans être saturés en tels ou tels concepts déterminés : c'est ainsi que, comme l'écrit Richir, l'horizon de la vie du sujet s'étend, «à perte de vue », à d'autres dans cette phénoménalisation. En ce sens, on pourrait dire que, dans la lecture richirienne, le jeu de l'imagination joue un rôle de phénoménalisation des phénomènes dans laquelle ils se croisent de façon libre, et non déterminée, les uns avec les autres ${ }^{4}$.

\footnotetext{
${ }^{1}$ Ibid., p. 102.

2 Dans Phantasia, imagination, affectivité. Phénoménologie et anthropologie phénoménologique (Grenoble, Jérôme Millon, coll. « Krisis », 2004), Richir approfondira cette force ludique, inhérente à l'imagination (phantasia «perceptive » dans ce contexte-là), à partir de la notion d' « objet transitionnel » que Donald Winnicott présente dans Jeu et Réalité, et dévoilera la couche la plus primordiale de la forme intersubjective qu'il appellera « interfacticité transcendantale ».

${ }^{3}$ M. Richir, «L'origine phénoménologique de la pensée », art. cit., p. 104.

${ }^{4}$ Ainsi Richir souligne la force qu'a l'imagination de modifier l'individu pour l'ouvrir à d'autres phénomènes. Cette tentative est aussi manifeste dans son interprétation de la «phantasia (Phantasie) » husserlienne. Car il souligne moins sa fonction contribuant à la réduction eidétique, à savoir la présentation du caractère unitaire de l'eidos du vécu intentionnel, que sa force de modification en tant que telle. Cf. M. Richir, Phantasia, imagination, affectivité. Phénoménologie et anthropologie phénoménologique, Grenoble, Jérôme Millon, coll. « Krisis », 2004, p. 81.
} 


\section{Imagination et humanisation}

\subsection{L'imagination dans le sublime kantien}

Dans les discussions précédentes, nous avons envisagé l'imagination kantienne, et sa lecture richirienne, exclusivement dans l'optique du jugement réfléchissant portant sur l'expérience esthétique, à savoir le " beau ». Pourtant, on ne pourrait pas manquer d'aborder un autre type d'expérience sur lequel portent le jugement réfléchissant et l'imagination : à savoir, l'expérience du « sublime».

Kant explique ce moment de «sublime " en le mettant en contraste avec le «beau »: " Le beau de la nature concerne la forme de l'objet, qui consiste dans la limitation ; en revanche, le sublime pourra être trouvé aussi en un objet informe (formlos) ${ }^{1}$. L'imagination mise en jeu dans le jugement esthétique portant sur le beau vise à figurer l'objet dans telle ou telle « forme », sans pourtant avoir recours aux concepts préétablis. Par contre, l'imagination en jeu au moment de sublime porte sur les expériences ou les moments que l'activité du sujet ne saurait formuler dans sa faculté cognitive. Celle-ci s'attache alors non à telle ou telle forme sensible de ses expériences, mais tente d'avoir rapport à ce qui n'apparaitrait jamais en substance dans sa sensibilité, c'est-à-dire le « supra-sensible (übersinnlich) $»^{2}$.

Conceptuellement, ce moment de sublime est divisé par l'auteur de la troisième critique entre le «sublime mathématique ${ }^{3}$ et le «sublime dynamique de la nature ${ }^{4}$. Dans le cas du premier, l'imagination et son jeu amènent le sujet à s'approcher de ce qui est « absolument grand (schlechthin gro $\beta) »^{5}$. La grandeur de celui-ci dépasse «toute comparaison» possible pour l'entendement humain, ainsi que la pyramide aux yeux des hommes au $18^{\mathrm{e}}$ siècle, selon l'exemple kantien ${ }^{6}$. Le sujet ne pourrait avoir alors d'autre prédicat ou énoncé qu' «absolument grand» pour expliquer le caractère grandiose de ce moment.

Le deuxième, «sublime dynamique », vient de l'esprit humain affecté et faisant face aux spectacles brutaux de la nature. Il ne s'y agit pas de la nature en tant qu'objet de la science naturelle mais de celle qui affecte

\footnotetext{
${ }^{1}$ E. Kant, Kritik der Urteilskraft, Ak. V, p. 244, trad. fr., p. 118.

${ }^{2}$ Ibid., p. 254, trad. fr., p. 133.

${ }^{3}$ Ibid., $\S \S 25-27$.

${ }^{4}$ Ibid., $\$ \S 27-29$.

${ }^{5}$ Ibid., p. 248, trad. fr., p. 123.

${ }^{6}$ Ibid., p. 252, trad. fr., p. 129.
} 
fortement, et même violemment, l'esprit du sujet et va jusqu'à lui faire éprouver de la "peur ${ }^{1}$. Un des fameux exemples kantiens précise tout cela :

Des roches se détachant audacieusement et comme une menace sur un ciel où d'orageux nuages s'assemblent et s'avancent dans les éclairs et les coups de tonnerre, des volcans en toute leur puissance dévastatrice, les ouragans que suit la désolation, l'immense océan dans sa fureur, les chutes d'un fleuve puissant, etc., ce sont là choses qui réduisent notre pouvoir de résister à quelque chose de dérisoire en comparaison de la force qui leur appartient ${ }^{2}$.

Le sublime dynamique que suscitent la nature et son aspect violent révèle à l'esprit du sujet une sorte d'impuissance à trouver le moyen d'y résister. Cependant, selon Kant, l'impuissance ne provoquerait jamais l' « angoisse » de la mort ou n'y laisse pas de traumatisme pathologique. Car l'angoisse provoquée au moment du sublime dynamique est décrite par lui comme «crainte respectueuse (Ehrfurcht) » ${ }^{3}$. Si l'imagination au moment de sublime amène le sujet à s'approcher des aspects grandioses, et même " sans limite (grenzlos)», de la nature, l'angoisse qu'il éprouve des choses démesurées le transpose dans un moment d' "humanité » ${ }^{4}$ au cours duquel il se met à éprouver un sentiment respectueux («crainte respectueuse ») à l'égard des choses se trouvant au-delà de la limite de ses expériences.

\subsection{La liberté phénoménologique et son paradoxe dans l'imagination}

La spécificité de l'imagination au moment de sublime consiste, selon la discussion kantienne, en ceci que sa mise en jeu se met à entrer en conflit avec la Raison :

Mais l'esprit [Gemüt] se sent grandir dans sa propre estime, si en cette contemplation [à savoir, celle des aspects violents de la nature, "masses montagneuses sans forme, entassées les unes sur les autres en un sauvage désordre, avec leurs pyramides de glace, etc. »] il s'abandonne, sans prêter attention à la forme de ces choses, à l'imagination et à la raison, qui ne fait

${ }^{1}$ Ibid., p. 260, trad. fr., p. 141.

${ }^{2}$ Ibid., p. 261, trad. fr., p. 142.

${ }^{3}$ Ibid., p. 264, trad. fr., p. 146.

${ }^{4}$ Ibid., p. 262, trad. fr., p. 143. 
qu'élargir l'imagination à laquelle elle se trouve liée [...] et si alors il trouve la puissance de l'imagination inférieure aux Idées de la raison ${ }^{1}$.

Au moment de sublime comme dans le cas du « beau », l'esprit (Gemüt) du sujet se trouve affecté et ému. Il est alors tellement affecté que l'imagination se dirige vers l'«appréhension» de la chose qui n'a pas de forme. Pour expliquer tout cela du côté de la Raison, celle-ci laisse alors l'imagination libre de se grandir; en bref, elle devient, pour sa part, «indéterminée (unbestimmt) $»^{2}$ en renonçant à régler la mise en jeu excessive de l'imagination à l'intérieur de ses idées régulatrices. Corrélativement à tout cela, la force de l'imagination est élevée jusqu'en ce qui est «illimité (Grenzlosigkeit) $»^{3}$.

C'est pour cette raison que Kant va jusqu'à dire qu' « imagination et raison [...] produisent [la «finalité subjective»] par leur conflit » ${ }^{4}$. D'un côté, il s'agit de l'idée régulatrice de la Raison; mais de l'autre, l'imagination y échappe dans sa pleine liberté et se laisse étendre dans son caractère illimité. C'est dans ces deux mouvements opposés l'un à l'autre que, au moment de sublime, l'imagination est forcée d'entrer en conflit avec la Raison. Tandis que l'imagination mise en jeu sur le plan du «beau»se trouve en relation harmonieuse avec l'entendement dans la " finalité sans fin », celle qui concerne le sublime laisse grandir l'esprit du sujet en l'affectant intensément.

C'est notamment à cette imagination kantienne, imagination mise en jeu en deçà des idées régulatrices de la Raison, que Richir porte attention pour élaborer sa propre phénoménologie de l'Einbildungskraft. Dans son ouvrage intitulé Phénoménologie et institution symbolique ${ }^{5}$, il y trouve une certaine liberté humaine, dont il précise le caractère phénoménologique. L'auteur en dit :

Visant à englober l'informe du phénomène qui lui échappe (ce qu'elle ne peut, pour nous, que dans le pro-jet d'une schématisation ou d'une phénoménalisation en langage), l'imagination se sent au bord de sa perte, menacée, en quelque sorte, dans la « libéralité », c'est-à-dire, dirons-nous, dans ce qui fait sa liberté phénoménologique. Enfin, cet excès, cette transcendance pour

${ }^{1}$ Ibid., p. 256, trad. fr., p. 135.

${ }^{2}$ Ibid., p. 244, trad. fr., p. 118.

${ }^{3}$ Ibid., p. 257, trad. fr., p. 122.

${ }_{5}^{4}$ Ibid., p. 258, trad. fr., p. 138.

${ }^{5}$ M. Richir, Phénoménologie et Institution symbolique. Phénomènes, temps et êtres II, Grenoble, Jérôme Millon, coll. « Krisis », 1988. 
l'imagination, à savoir pour la schématisation phénoménologique, ne l'est pas pour l'idée de la Raison, c'est-à-dire, ici pour le transphénoménal, qui est cela même qui, pour Kant, pousse en fait l'imagination à schématiser, fût-ce sans succès ${ }^{1}$.

Richir qualifie de «liberté phénoménologique » l'imagination kantienne au moment du sublime, en tant qu'elle se dérobe aux idées régulatrices de la Raison. C'est au sein de cette « liberté » que l'imagination se met à « viser », à appréhender ce qui dépasse l'entendement et le concept de sujet : à savoir, l'«absolument grand», dans le cas du sublime mathématique, et ce qui est « sans limite », s'il s'agit du dynamique. Si on explique tout cela suivant la remarque richirienne ci-dessus, le sujet s'approche, au sein de la fonction de « schématisation » inhérente à l'imagination, de ce qui ne serait jamais figurable dans son expérience et dans son intuition; alors, sa conscience caractérisée par l'imagination se gonfle de façon hyperbolique dans la «transcendance " et, dès lors, arrive à s'attacher à une part "informe" de ses expériences. Et celle-ci n'est, selon la définition richirienne, rien d'autre que les éléments essentiels $d u$ "phénomène" au sens phénoménologique. Comme dans le cas du «beau », la liberté de l'imagination en jeu au moment de sublime a ainsi pour fonction de phénoménaliser telles ou telles expériences du sujet dans leur phénoménalité.

En outre, la spécificité phénoménologique de la phénoménalisation au moment de sublime consiste à conduire les expériences du sujet au bord de leur limite au bout de laquelle elles seraient absorbées dans la nature déchaînée. En effet, dans la citation ci-dessus, Richir ne manque pas d'indiquer le caractère paradoxal de la "liberté phénoménologique », sans se contenter de préciser son caractère libre et transcendant. La mise en jeu de l'imagination au moment de sublime est tellement excessive (" excès ») que ce mouvement illimité, et même en conflit avec la Raison, est forcé de s'approcher dangereusement de la «perte » de sa liberté. Pour paradoxal que ce soit, si l'imagination au moment de sublime se délivre des idées régulatrices de la Raison, son caractère excessivement libre et illimité l'amène ellemême à la crise de sa propre disparition, puisque sa libre mise en jeu en tant que telle ne peut se stabiliser sur rien; c'est à cause de sa propre "libéralité », et non de tels ou tels facteurs externes, que son libre jeu se trouve menacé inévitablement par sa disparition. C'est ainsi que, dit Richir, l'«excès» de l'imagination se retourne, au bout de sa mise en jeu

${ }^{1}$ Ibid., p. 100. 
excessivement libre, sur le « défaut $»^{1}$ ou la "lacune $»^{2}$ de son accomplissement. Tout cela est appelé par lui « échec de phénoménalisation $»^{3}$. On peut comprendre dès lors que, en discutant soigneusement de l'imagination kantienne, Richir tente d'en dégager l'aspect paradoxal : à savoir, l'imagination dédoublée, à son origine, entre sa mise en jeu excessive et la crise de sa disparition ("échec ») à cause de sa liberté.

\subsection{L'humanisation dans l'imagination}

Loin d'être un moment négatif ou pessimiste, l'aspect paradoxal de ce double mouvement, inhérent au libre jeu de l'imagination, constitue lui-même l'humanité de l'homme au sein des phénomènes. En effet, dit Richir, le phénomène qui se phénoménalise par l'exercice de l'imagination est exactement le «phénomène humain ${ }^{4}$. Cela signifie que, si l'imagination richirienne se fait foncièrement à la limite entre sa mise en jeu excessive et la crise imminente de sa disparition, elle-même et sa phénoménalisation amènent l'homme à assister aux aspects paradoxaux, et même énigmatiques, de ses vécus ; c'est-à-dire de l' « excès » et le « défaut » de son existence. Tout cela apparaît lorsque l'auteur tente de trouver dans le sublime kantien une certaine genèse du sens phénoménologique :

D'une certaine manière, si le sublime donne le vertige, c'est qu'il laisse sans voix, ouvrant certes la voix à elle-même, mais au bord du gouffre ou de l'abîme ouvert par l'apeiron des phénomènes, donc ouvrant du même coup le phénomène de langage aux lacunes qui surgissent comme impossibles à combler de sa phénoménalité. C'est donc tout le contraire de la finalité sans fin des phénomènes qui ouvre à elle-même la finalité du phénomène de langage ${ }^{5}$.

Le moment où le sens s'exprime dans sa structure génétique est défini, par Richir, comme "phénomène de langage». Le lieu typique de ce moment génétique est caractérisé par l'auteur sous les termes d' «abîme» ou de "gouffre». Faisant face aux moments de sublime, l'homme se trouve pris d'un «vertige». À mesure que le phénomène s'y phénoménalise de façon

\footnotetext{
${ }^{1}$ Ibid., p. 94.

${ }^{2}$ Ibid., p. 100.

${ }^{3}$ Ibid., p. 100.

${ }^{4}$ Ibid., p. 93.

${ }^{5}$ Ibid., p. 97.
} 
illimitée (« apeiron »), l'homme se sent frissonner à un tel moment abyssal. Il ne peut plus alors trouver ses propres « voix » susceptibles de thématiser cette expérience vertigineuse dans tels ou tels concepts. Pourtant, loin de s'y absorber, sa conscience est affectée par cette expérience "sans voix » et amenée à chercher son « sens » dans un tel état lacunaire. En considérant de cette manière la genèse du sens au moment de sublime, Richir souligne le moment abyssal où l'homme et sa conscience s'approchent, dans l'abîme, du sens de ses vécus. Selon Richir, si la conscience humaine se trouve dans la structure génétique du "phénomène de langage ", celui-ci amène celle-ci à s'approcher du sens de ses expériences dans la situation limite (l' "abîme » ou le « gouffre »), au-delà de laquelle elle s'anéantirait. Et tout cela signifie que l'humanité de l'homme, à savoir le "phénomène humain », se phénoménalise entre la mise en jeu de l'imagination et le moment abyssal où est érodée inévitablement sa conscience intentionnelle.

La spécificité de l'approche richirienne du sens sera détaillée par sa mise en contraste avec la théorie husserlienne de la « signification». Husserl considère comme processus nécessaire de la « signification » la " plénitude » (ou le « remplissement») de la conscience intentionnelle par l'intuition : «les actes qui lui [le phénomène physique] donnent la signification, et éventuellement, sa plénitude intuitive (anschauliche Fülle), et où se constitue sa référence à une objectité exprimée ${ }^{1}$. Le fait que la «signification» s'exprime comme essence des vécus du sujet veut dire que l'intention de signification de celui-ci, vide en elle-même, arrive à être remplie par l'intuition. Les «actes» de sa conscience dans cette "plénitude» ont pour effet de transformer ou d' «animer (beleben) » ${ }^{2}$ les événements qui étaient juste physiques (les mots, les voix, etc.) en des "référents » auxquels ses vécus s'attachent dans la structure intentionnelle.

$\mathrm{Au}$ contraire de la théorie de "signification » husserlienne, le sens se fait, selon Richir, au moment où il n'y aurait plus moyen d'animer les évènements physiques par les actes de conscience. Car ces phénomènes physiques (les aspects violents de la nature) sont eux-mêmes transfigurés en « supra-sensibles » (« apeiron des phénomènes ») dans l'esprit du sujet. Loin

\footnotetext{
${ }^{1}$ E. Husserl, Logische Untersuchungen, zweiter Band (II/1) : Untersuchungen zur Phänomenologie und Theorie der Erkenntnis (1900), Tübingen, Max Niemeyer, 1993, cite d'après Husserliana Band XIX/1, p. 37; trad. fr. Hubert Elie, Arion L. Kelkel et René Schérer, Recherches logiques, tome 2 : Recherches pour la phénoménologie et la théorie de la connaissance, première partie : Recherches I et II, Paris, PUF, coll. « Épiméthée », 1961, p. 43.

${ }^{2}$ Ibid., p. 38, trad. fr., p. 44.
} 
d'être remplie par l'intuition de l'objet, la conscience intentionnelle devient elle-même vertigineuse devant l'aspect infigurable, et supra-sensible, de ses expériences sans trouver aucune " voix » susceptible de l'indiquer comme corrélat de ses actes. C'est en ce sens que Richir souligne l' «abîme» ou le « gouffre » pour préciser la genèse du sens phénoménologique. Et c'est dans un tel creux ou une épreuve bouleversant l'esprit humain, et non suivant la plénitude de la conscience intentionnelle, que la vie humaine peut accéder au sens de ses vécus, à savoir au " phénomène de langage ».

Si on considère le rôle de l'imagination dans ce contexte, on pourrait dès lors comprendre que sa mise en jeu et son caractère excessif jouent un rôle pour l'humanisation de l'homme au moment de sublime. Elle est, chez Richir comme chez Kant, la tentative d' " appréhender» la part « informe » de l'expérience, quoiqu'elle soit destinée à l'« échec » à cause de sa libéralité. Cela revient à dire que, si cette " appréhension » imaginaire n'était pas mise en jeu pour saisir, "fût-ce sans succès », le moment abyssal et informe du sublime qui bouleverse l'esprit humain, la conscience de celui-ci resterait pour toujours lacunaire et dès lors ne pourrait jamais s'approcher du sens de sa vie. Le sens phénoménologique est, selon Richir, mis en genèse entre le moment vertigineux du sublime et la libre mise en jeu de l'imagination qui tente de l'appréhender. Si on explique ce sens phénoménologique notamment à partir de la mise en jeu de l'imagination, c'est à travers sa force d'appréhender l'«informe» que la vie humaine peut éviter de s'absorber dans l'abîme du moment vertigineux et dès lors garder pour elle-même sa propre humanité. C'est en ce sens qu'il nous est possible d'affirmer que l'excès de l'imagination fait partie intégrante de l'humanisation de l'humanité dans les "phénomènes humains ».

\section{Imagination et institution symbolique de la Raison}

\subsection{Le surgissement et l'institution du soi}

Ainsi, pour dégager sa portée phénoménologique, Richir tente de souligner, comme l'a fait Hannah Arendt ${ }^{1}$, le caractère spécifique de l'imagination

\footnotetext{
${ }^{1}$ En discutant de la troisième critique kantienne dans sa conférence, Hannah Arendt distingue rigoureusement le jugement réfléchissant faisant sortir l'imagination et la loi morale fondée sur la Raison pratique, et apprécie le premier dans la théorie kantienne du politique. C'est ainsi qu'elle va jusqu'à dire : «Car le jugement portant sur le particulier - Ceci est beau, Ceci est laid ; Ceci est bien, Ceci est mal — n'a pas sa place dans la philosophie morale de Kant. Le jugement n'est pas la raison
} 
kantienne, et sa libre mise en jeu, elle-même irréductible aux idées régulatrices de la Raison. Cependant, selon sa conception, notamment au moment de Phénoménologie et institution symbolique et Du sublime en politique ${ }^{1}$, le fonctionnement de la Raison n'est pas forcément nié mais nous permet de voir un autre registre de la phénoménalité du phénomène humain. Il l'appelle « institution symbolique » de la vie du sujet. Richir repère ce moment instituant, et son caractère phénoménologique, dans le processus de surgissement du soi au moment de sublime kantien. La manière dont le soi surgit au moment de sublime est précisée, du côté de la discussion kantienne, au § 28 de la troisième critique :

Tout de même sa [«nature »] force irrésistible nous fait connaître en tant qu'êtres de la nature notre faiblesse physique (physische Ohnmacht), mais en même temps elle dévoile une faculté (Vermögen), qui nous permet de nous considérer comme indépendants par rapport à elle, et une supériorité sur la nature, sur laquelle se fonde une conservation de soi-même (Selbsterhaltung) toute différente de celle qui est attaquée par la nature $[\ldots]^{2}$.

Selon Kant, les aspects violents de la «nature» au moment du sublime dynamique conduisent le sujet à éprouver sa propre «faiblesse ». Cependant, cela ne signifie pas que ce sujet, en tant qu'être impuissant, s'incline luimême devant la nature déchaînée. Car, selon la remarque kantienne cidessus, si le sublime est un moment $\mathrm{d}^{\prime}$ " humanité $»^{3}$, le sujet faisant face à la crise de sa disparition tente de se tenir soi-même pour préserver sa propre existence ; il se met à «conserver» son identité et sa dignité sans se laisser être absorbé dans les scènes vertigineuses de la nature déchaînée, quoiqu'elles soient des «petites choses ${ }^{4}$ par rapport à la puissance grandiose et même terrifiante de la nature. C'est à ce moment de réaction du sujet contre la nature que l'être humain se met à prendre conscience de lui-même, de son ipséité et de sa finitude. Et, suivant cette prise de conscience de soi,

pratique ; la raison pratique "raisonne" et me prescrit ce que je dois et ne dois pas faire; elle instaure la loi et est identique à la volonté. La volonté énonce des commandements et parle à l'impératif. À l'inverse, le jugement naît d'un "plaisir seulement contemplatif ou satisfaction inactive [untätiges Wohlgefallen]" ") (H. Arendt, Lectures on Kant's Political Philosophy, op. cit., p. 15, trad. fr., p. 32-33).

${ }^{1}$ M. Richir, Du sublime en politique, Paris, Payot, coll. " Critique de la politique », 1991.

${ }^{2}$ E. Kant, Kritik der Urteilskraft, Ak. V, p. 261, trad. fr., p. 143.

${ }^{3}$ Ibid., p. 262, trad. fr., p. 143.

${ }^{4}$ Ibid., p. 262, trad. fr., p. 143. 
ses activités se mettent à rejoindre les idées de la Raison avec lesquelles l'imagination au moment de sublime était jusqu'alors en conflit. Richir considère ce surgissement du soi comme un moment instituant de la vie du sujet :

Puissance terrifiante de destruction, qui doit bien habiter, quelque part, l'instituant symbolique, mais qui, ce qui est capital, suscite, par son recul même dans le sublime, notre force, notre pouvoir, qui n'est pas lui-même nature, mais qui est aussi au-dessus de notre appréhension empirique de la vie. Dans nos termes, cela signifie que cette puissance pointe ou épingle la force d'institution symbolique qu'il y a en nous [...] $]^{1}$.

Effrayé par la «puissance terrifiante» de la nature au moment du sublime dynamique, l'homme en vient à prendre conscience de la finitude de son être. Cette prise de conscience dans les idées de la Raison a pour effet, selon la remarque ci-dessus de Richir, d'annoncer un moment instituant de la vie du sujet : c'est le moment où l'homme, ses activités et même ses vécus se mettent à être institués symboliquement. Car son être y est tout d'abord délimité entre la vie et la mort à mesure qu'il se met à prendre conscience de son impuissance devant la violence de la nature. Il arrive ensuite à se distinguer de la nature qui le terrifie. Son statut se met à se déplacer vers la nature en nous ("notre force») et, dès lors, à se distinguer de la nature externe. Cette série de changements du sujet indique que son être se trouve, à ce moment instituant, découpé entièrement entre la nature et la culture. Le moment instituant de la Raison a ainsi pour effet de découper la vie humaine tant entre la vie et la mort qu'entre la nature et la culture.

Il faut alors préciser le fait que ce moment instituant soit qualifié par Richir de «symbolique » et non effectif. Car la vie du sujet est instituée par lui de telle façon qu'il ne pourrait comprendre dans quel processus « empirique » et observable il devient conscient de sa finitude et de sa propre différence d'avec la nature. Le moment instituant de la Raison est « symbolique », et non empirique, dans la mesure où le sujet est institué à son insu. Tout cela signifie que, si l'imagination en conflit avec la Raison dans sa pleine liberté conditionne phénoménologiquement la phénoménalisation du "phénomène humain », la Raison tente, dans cette opposition, de délimiter et de stabiliser la liberté humaine et son caractère excessif. Richir dégage ainsi à partir de l'imagination kantienne l'humanité se trouvant entre deux registres radicalement opposés du phénomène, à savoir ce qui est du « phénoménologique » et du « symbolique ».

${ }^{1}$ M. Richir, Phénoménologie et Institution symbolique, op. cit., p. 117. 


\subsection{La rencontre du phénoménologique et du symbolique}

Corrélativement à l'institution de la vie humaine, l'imagination, sa libre mise en jeu et son caractère excessif y sont destinés à être stabilisés en étant récupérés dans les idées de la Raison. Richir trouve dans ce processus de stabilisation le caractère paradoxal, et même énigmatique, de la vie humaine qui serait appelée par lui « rencontre phénoménologique » :

C'est donc, en ce sens, une lacune en phénoménalité dans le phénomène de langage, en tant qu'échec de la phénoménalisation de langage, qui ne peut paraître au champ phénoménologique qu'en tant qu'abîme ou gouffre en lequel la schématisation phénoménologique en langage est sur le point de se perdre, et est dans cette mesure incitée à se reprendre, mais poussée par l'idée de la Raison comme lieu symboliquement instituant un sens en tant que pro-jet de re-schématisation en langage de la ou des lacunes en phénoménalité ${ }^{1}$.

Comme on l'a vu, le caractère excessivement libre de l'imagination la destine, à cause de sa propre "libéralité ", à être conduite au bord de sa «perte ». Selon la remarque ci-dessus de Richir, cela ne signifie pas simplement une disparition de l'imagination mais sa transposition dans un autre registre du phénomène, à savoir le «lieu symboliquement instituant un sens ».

Lorsque la fonction schématique (le « schématisme ») de l'imagination faillit se perdre jusqu'au fond de son propre "abîme », elle devient, à son tour, récupérée par l'idée de la Raison. Tout cela est nommé par Richir «reschématisation ». Celle-ci tente d'exprimer à nouveau un "sens». Mais celui-ci n'est plus le même que celui observé au moment de la première mise en jeu de l'imagination (le "schématisme »). Car il se fait, à son tour, dans le cadre des idées de la Raison. Certes, la conscience intentionnelle de l'homme vise le sens de ses vécus dans le cadre de la «re-schématisation». Mais, le sens qu'elle vise en "pro-jet» se glisse, à son insu, de celui qui était en genèse vivement dans la libre mise en jeu de l'imagination à celui stabilisé par l'institution de la Raison. Richir dégage ainsi, avec sa conception de « reschématisation ", le changement dynamique de registre du sens phénoménologique et la situation paradoxale des activités humaines.

Ce changement de registre atteste que la vie du sujet, son esprit affecté dans le sublime et l'imagination qui y est mise en jeu excessivement se stabilisent également dans l'« institution symbolique »; la «liberté phéno-

${ }^{1}$ Ibid., p. 100. 
ménologique» est alors remplacée, à l'insu du soi, par la «liberté symbolique $»^{1}$. Le statut de la dernière est, par nature, tant ambivalent qu'énigmatique. Car, selon la remarque de Richir dans $D u$ sublime en politique, ce registre symbolique a pour fonction de stabiliser la forme intersubjective de la vie humaine. Analysant le même texte et le même contexte kantien, l'auteur écrit

[...] c'est la Loi, précisément, de la fidélité à l'énigme de ma condition comme condition humaine, celle que je suis amené à partager avec les autres, en tant donc qu'elle résonne en écho à l'énigme insondable et imprépensable (dans le concept ou l'intuition) de l'Autre'2 .

Selon Kant, la «Loi » n'est pas un objet apparaissant dans le champ intuitif ni un concept susceptible d'être déterminé dans l'entendement humain, mais juste une instance «universelle». C'est ainsi que, selon la remarque cidessus de Richir, la récupération de la libre mise en jeu de l'imagination dans les idées de la Raison - Raison pratique (la « Loi » morale) dans ce contexte - non seulement sauve le sujet de la conséquence chaotique ( «abîme ») de l'excessive liberté de l'imagination mais aussi l'amène à partager sa « condition humaine » avec d'autres sujets. En étant ainsi récupéré par les idées de la Raison, le sujet se trouve alors face à l' " énigme » de son rapport à d'autres. Plus précisément, c'est dans cette énigme du moment instituant de la Raison qu'il est ouvert, d'une façon "imprépensable », à l'altérité ("Autre ») d'autres sujets et commence à la respecter sans se renfermer dans son ipséité. En ce sens, loin de limiter sa discussion à l'aspect impératif de la «Loi » morale kantienne, Richir y affirme son aspect instituant qui relie l'être humain à la communauté intersubjective.

Cependant, c'est aussi à ce moment « instituant» de la vie du sujet que l'on peut trouver l'aspect ambivalent de l'institution symbolique de la Raison: d'un côté, elle initie les êtres humains à leur communauté intersubjective dans son universalité mais, de l'autre, l'imagination et sa liberté phénoménologique («phénoménalisation» selon la terminologie richirienne) disparaissent à tel point que la forme « quasi-sauvage » de l'intersubjectivité se stabilise dans la communauté des " égaux $»^{3}$ qui se trouveraient ensemble devant l'universalité d'une seule et même « Loi » morale.

\footnotetext{
${ }^{1}$ Ibid., p. 100.

${ }^{2}$ M. Richir, Du sublime en politique, op. cit., p. 62.

${ }^{3}$ Ibid., p. 63.
} 
Tout cela est résumé par la remarque suivante de l'auteur: «Le symbolique rencontre le phénoménologique $»^{1}$. Dès que l'imagination est mise en jeu au moment du sublime, son caractère excessivement libre devrait se mettre à être récupéré simultanément par l'institution symbolique (le « non phénoménologique ») puisqu'elle est destinée, par nature, à l'« échec » que cause sa radicale libéralité. Le moment instituant y intervient alors de telle façon que le sujet ne pourrait alors comprendre comment et dans quel processus sa liberté dans la mise en jeu de l'imagination, et l'intersubjectivité « quasi-sauvage » qui n'a recours à aucun procédé intermédiaire, se glisse dans la communauté instituée des "égaux ». Tout cela signifie que l'imagination au sens phénoménologique se trouve être mise en jeu, à un seul et même moment, entre deux mouvements opposés l'un à l'autre; entre l'excessive liberté qui fait suite au moment de sublime, c'est-à-dire humanisation, et son éventuelle récupération possible qui la stabilise dans l'institution de la Raison.

\section{Conclusion}

En s'approchant de l'Einbildungskraft kantienne dans la troisième critique, Richir tente de la considérer comme "phénoménalisation » des phénomènes. Cette approche phénoménologique lui permet de décrire l'aspect paradoxal de la vie humaine qui est dédoublée entre l'humanisation et son éventuelle fixation dans l'institution. Tout cela signifie que, loin de souligner simplement la fonction intermédiaire de l'imagination, son approche vise à mettre en perspective globale la genèse (la liberté phénoménologique) et la conséquence (institution) de la mise en jeu de l'imagination. C'est à travers la «rencontre» de ces deux registres que l'aspect "énigmatique» de la phénoménalisation du «phénomène humain» se manifeste dans la vie humaine. De cette manière, l'Einbildungskraft kantienne inspire les idées phénoménologiques de Richir. Il nous est dès lors permis d'affirmer que, grâce à cette approche phénoménologique, l'Einbildungskraft présentée par Kant au $18^{\mathrm{e}}$ siècle rejoint, à son tour, la tradition de la phénoménologie transcendantale.

${ }^{1}$ M. Richir, Phénoménologie et Institution symbolique, op. cit., p. 112. 


\section{Bibliographie}

Arendt Hannah, Lectures on Kant's Political Philosophy, edited and with an interpretive essay by R. Beiner, The University of Chicago Press, 1982; trad. fr. M. Revault d'Allonnes, Juger. Sur la philosophie politique de Kant, Paris, Seuil, 1991.

Husserl Edmund, Logische Untersuchungen, zweiter Band (II/1) : Untersuchungen zur Phänomenologie und Theorie der Erkenntnis (1900), Tübingen, Max Niemeyer, 1993; trad. fr. Hubert Elie, Arion L. Kelkel et René Schérer, Recherches logiques, tome 2 : Recherches pour la phénoménologie et la théorie de la connaissance, première partie: Recherches I et II, Paris, PUF, coll. « Épiméthée », 1961.

Husserl Edmund, Ideen zu einer reinen Phänomenologie und phänomenologischen Philosophie. Erstes Buch: Allgemeine Einführung in die reine Phänomenologie (1913), Halle, Max Niemeyer; trad. fr. Paul Ricoeur, Idées directrices pour une phénoménologie pure et une philosophie phénoménologique, Paris, Gallimard, coll. « Tel », 1950.

Kant Emmanuel, Kritik der Urteilskraft (1790), cité d'après la version de la Königlich Preußischen Akademie der Wissenschaften (Ak.); trad. fr. A. Philonenko, Critique de la faculté de juger, Paris, J. Vrin, 1993.

Richir Marc, «L'origine phénoménologique de la pensée », La liberté de l'esprit, $\mathrm{n}^{\circ}$ 7, Paris, Balland, 1984, p. 63-107.

Richir Marc, Phénoménologie et Institution symbolique. Phénomènes, temps et êtres II, Grenoble, Jérôme Millon, coll. « Krisis », 1988.

Richir Marc, Du sublime en politique, Paris, Payot, coll. « Critique de la politique », 1991.

Richir Marc, Phantasia, imagination, affectivité. Phénoménologie et anthropologie phénoménologique, Grenoble, Jérôme Millon, coll. « Krisis », 2004. 